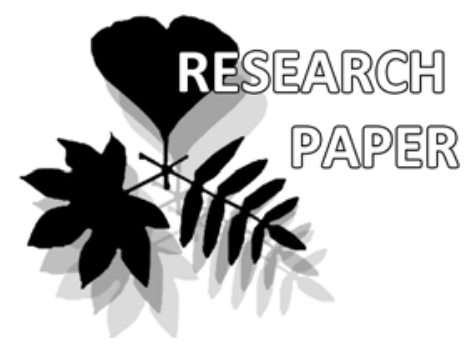

\title{
A new checklist of the liverworts and hornworts of Georgia (Caucasus)
}

\author{
Vadim Bakalin ${ }^{1 *}$, Ketevan Tigishvili ${ }^{2} \&$ George Arutinov ${ }^{2}$
}

Vadim Bakalin ${ }^{1 *}$

e-mail: vabakalin@gmail.com

Ketevan Tigishvili ${ }^{2}$

e-mail: ketevan.tigishvili@gmail.com

George Arutinov ${ }^{2}$

e-mail: arutinovgeorge@yahoo.com

${ }^{1}$ Botanical Garden-Institute FEB RAS, Vladivostok, Russia

${ }^{2}$ Institute of Botany of Ilia State

University, Tbilisi, Georgia

* corresponding author

Manuscript received: 10.02 .2016

Review completed: 25.03.2016

Accepted for publication: 04.04.2016

Published online: 06.05.2016

\begin{abstract}
A B S T R A C T
The newly updated and revised checklist of Georgian liverworts and hornworts is provided. It records 172 species (including 171 native and one introduced, once recorded and currently probably extinct), with each species annotated by synonyms previously used in Georgia, data on distribution within administrative subunits of Georgia, literature reference and presence of specimens examined in herbaria TBI and VBGI.
\end{abstract}

K e y w o r d s : Hepaticae, liverworts, Anthocerotae, hornworts, Georgia, Caucasus, checklist

\section{P E 3 Ю M E}

Бакалин В., Тигишвили К., Арутюнов Г. Новый чеклист печеночников и антоцеротовых Грузии (Кавказ). Состав ее чеклист печеночников и антоцеротовых Грузии, вкАючающий 172 вила (вкАючая 171 аборигенный виА и 1 случайно интродуцированный и в настоящее время, вероятно, «выпавший» из состава флоры). Каждый виА аннотирован сведениями о распространении в пределах аАминистративных единиц Грузии, ссылками на работы в которых этот виА приводился Аля страны и наличием образцов с территории Грузии в гербариях TBI и VBGI.

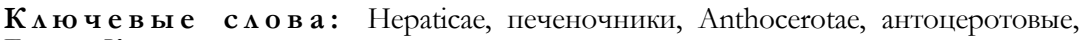
Грузия, Кавказ, чеклист
Georgia is the Caucasian country situated on the border between Asia and Europe and washed by the Black Sea in the west. It characterized by great differences in climatic conditions, varying from mild and wet warm temperate in the Colchis forests of Adjara to sub-continental cool temperate in the inner part. Whereas Georgia covers a relatively small area $\left(69000 \mathrm{~km}^{2}\right.$, including self-proclaimed South Ossetia and Abkhazia), the flora of 'higher' plants (mosses, liverworts, hornworts and angiosperms) of the land is rather diverse and comprises over 5000 species, 172 of which are hepatics (including both liverworts and hornworts). Unfortunately, Georgia has not had a checklist compiled in accordance with new nomenclature. Available checklists (although with the last published only one decade ago, Chikovani \& Svanidze 2004) are out of date and sometimes bear nomenclatural mistakes.

To fill this gap and to promote further studies of Georgian hepatics we provide a checklist based on all available sources and in accordance with modern taxonomy. To harmonize the distributional data from previous checklists (Chikovani 1986, Chikovani \& Svanidze 2004) and other sources was one of the most difficult tasks of the present work, since those authors used a so-called historical-geographic subdivision of Georgia that is not official and has unstable and not evident borders. In contrast, the present checklist follows the official current subdivision of Georgia for the first time (http://gov. ge/index. php?lang id=ENG\&sec_id=227\&info_id=31239 last access March 2016).

Although the flora of the Caucasus in general, and Georgia in particular, have long attracted attention, the first records of hepatics for this land were published by Kuntze and Regel (1887) based on L. Kaernbach's collections. The authors (l.c.) provided a list of six species based on F. Stephani's identifications, five of which were from Georgian Adjara: Anthoceros dichotomus Raddi (= Phaeoceros bulbiculosus), Chiloscyphus polyanthos Nees, Jungermannia sphaerocarpa Hook. (= Solenostoma sphaerocarpum), Radula commutata Gottsche (= R. complanata) and Reboulia hemisphaerica Raddi. Thereafter the greatest input to the knowledge of Georgian liverworts was made by E. Levier in collaboration with S. Sommier in the course of exploration for Caucasian plants. The hepatic material gathered by E. Levier was identified by F. Stephani along with several specimens collected by V. F. Brotherus and published in the widely known and still valuable "Enumeratio Muscorum Caucasi”" (Brotherus 1882). Here he cited 94 species of hepatics, 93 of which were recorded from Georgia.

In the next half century, rich in political crises, further studies of Georgian hepatics were impeded. Only two 
works that bore information on hepatic diversity were published in that time (Voronov 1914, Bush \& Bush 1936). They contributed a little in understanding the Georgian hepatic flora. Fortunately, the first three decades of the second half of 20 th century were very productive due to miscellaneous works, including local checklists, flora analyses and revision of some groups (Abramova \& Abramov 1953a, 1953b, 1979, Abramov et al. 1964, 1986, Chikovani 1972, 1973, Duda 1982, etc.). The great achievements in this field resulted in the publication of a checklist of Georgian liverworts and hornworts (Chikovani 1986). The latter provides data on distribution of 161 species including records of unclear origin, i. e., we were unable to find either herbarium specimens or original literature records for more than 10 taxa (Riccardia chamedryfolia, Isopaches bicrenatus, etc.). Similarly, the same taxa were mentioned under multiple names (Jungermannia tristis and then J. atrovirens) in the list. Only a few local papers appeared after the latter conspectus (Váňa \& Ignatov 1990, Tigischvili 2006, Kürschner et al. 2013, Bakalin \& Tigischvili 2013, Bakalin et al., 2013).

The last attempt to summarize data on the hepatics of Georgia was undertaken in 2004 with the publication "Checklist of Bryophyte Species of Georgia" by Chikovani \& Svanidze (2004). The latter represents a shortened version of conspectus published 18 years before (Chikovani 1986) with some inexplicable omissions. Nomenclature usage in the both mentioned checklists sometimes was unclear, as was mentioned by Bakalin \& Tigishvili (2013). Also Chikovani \& Svanidze (2004) stated in the abstract that they recorded 174 taxa of hepatics, but really listed in the checklist only 156 taxa (152 species) and overlooked even some previous indications of taxa in Chikovani (1986), but sometimes provided the same species under different names.

\section{RESULTS AND DISCUSSION}

The present checklist adds 20 species to the Chikovani \& Svanidze (2004) checklist and totals 172 species (including recently introduced and now probably extinct Nardia assamica). In our checklist, we mostly followed to the last checklist of Russian liverworts (Konstantinova et al. 2009) in both nomenclature and systematics. The synonyms are provided only if they were used in literature on Georgian hepatics. Each species is annotated by the names of administrative subunits where the species is recorded, literature references in which the record is based and presence of specimens of the species in TBI and/or VBGI. As it is evident from Table 1, the state of knowledge on the Georgian hepatic flora varies from region to region. The highest number

Figure 1 Administrative subdivision of Georgia (numbers indicate regions as they appear in Table 1 of taxa is recorded in Adjara (107 species), followed by Mtskheta-Mtianeti (89) and Shida Kartli (87). Other regions total from 53 to 77 taxa that, at least in some cases, should be far from reality.

The intricate question is how many taxa may be recorded as new for Georgia in further studies. As Chikovani (1978: 56) wrote (groundlessly ascribing this to Brotherus) "Caucasus being very rich by endemics of vascular plants did not support the same expectation [in hepatic flora]". This point of view may be fully applied particularly to Georgia as well. A recent comparison of hepatic floras of Manchuria and Caucasus (Bakalin 2014a) also showed a comparative poorness of Caucasian hepatic flora that may be explained by the complex of natural historical factors. Nevertheless, we believe that future research will add at least 20-25 species to the list of Georgian hepatics and therefore the known hepatic flora will reach approximately 200 species. In addition to the wetter areas of Abkhazia, Samgrelo, Guria and Adjara, where hepatic flora should be richer than it is now known, attention should also be paid to Kvemo Kartli and Kakheti.

Table 1. The administrative subdivisions of Georgia (excluding the city of Tbilisi) and the number of hepatic species recorded for each.

\begin{tabular}{|r|l|c|}
\hline No. & Region & $\begin{array}{c}\text { No. of species } \\
\text { recorded }\end{array}$ \\
\hline 1 & Abkhazia & 76 \\
\hline 2 & Samegrelo-Zemo Svaneti & 53 \\
\hline 3 & Guria & 53 \\
\hline 4 & Adjara & 107 \\
\hline 5 & Racha-Lechkhumi and Kvemo Svaneti & 77 \\
\hline 6 & Imereti & 60 \\
\hline 7 & Samtskhe-Javakheti & 66 \\
\hline 8 & Shida Kartli & 87 \\
\hline 9 & Mtskheta-Mtianeti & 89 \\
\hline 10 & Kvemo Kartli & 62 \\
\hline 11 & Kakheti & 64 \\
\hline
\end{tabular}

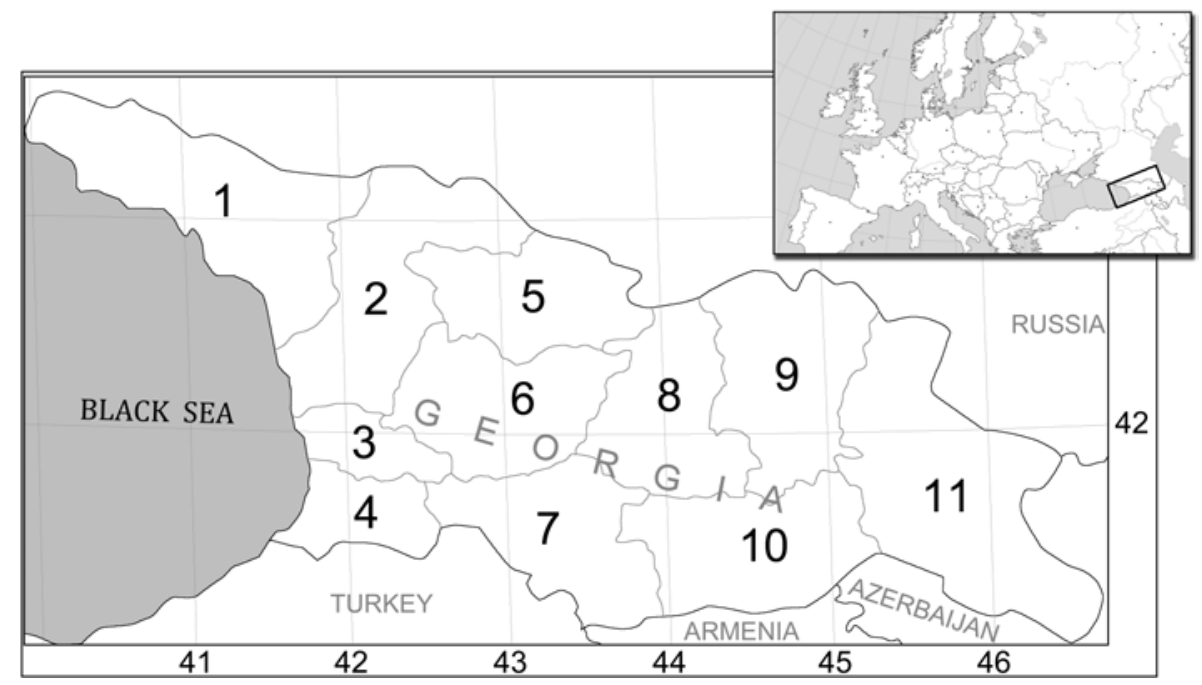




\section{LIST OF SPECIES}

\section{ANTHOCEROTOPHYTA}

Anthocerotaceae Dumort.

\section{Anthoceros L.}

A. cancasicus Steph. - Adjara [Abramov et al. 1966, Duda 1982, Chikovani \& Svanidze 2004, Bakalin et al. 2013; VBGI].

A. punctatus L. - Adjara [Abramov et al. 1966, Chikovani \& Svanidze 2004, Bakalin et al. 2013; VBGI].

\section{Phaeoceros Prosk.}

P. laevis (L.) Prosk. subsp. laevis (三 Anthoceros laevis L.) - Adjara [Abramov et al. 1966, Chikovani \& Svanidze 2004, Bakalin et al. 2013; VBGI.

P. carolinianus (Michx.) Prosk. - Adjara [Duda 1982].

Phymatoceros Stotler, W.T. Doyle et Crand.-Stotl.

P. bulbiculosus (Brot.) Stotler, W.T. Doyle et Crand.-Stotl. (= Anthoceros dichotomus Raddi) - Adjara [Brotherus 1892]

\section{MARCHANTIOPHYTA}

Blasiaceae H. Klinggr.

Blasia L.

B. pusilla L. - Adjara, Racha-Lechkhumi [Brotherus 1892 , Abramov et al. 1966, Chikovani 1986, Chikovani \& Svanidze 2004, Bakalin et al. 2013; VBGI.

Lunulariaceae H. Klinggr.

\section{Lunularia Adans.}

L. cruciata (L.) Dumort. ex Lindb. - Adjara [Abramov et al. 1966, Chikovani 1986, Chikovani \& Svanidze 2004, Bakalin et al. 2013; VBGI.

Marchantiaceae Lindl.

\section{Marchantia L.}

M. paleacea Bertol. - Abkhazia, Adjara, Guria, Imereti, Samegrelo [Brotherus 1892, Ladyzhenskava 1951, Abramova \& Abramov 1953b, Abramov et al. 1966, Chikovani 1986, Chikovani \& Svanidze 2004].

M. polymorpha L. s.l. - all regions [Brotherus 1892, Abramova \& Abramov 1953a, Abramov et al. 1963, 1964, 1966, Duda 1982, Chikovani \& Svanidze 2004, Bakalin et al. 2013; VBGI].

Preissia Corda

P. quadrata (Scop.) Nees (= Chomiocarpon quadratum (Scop.) Lindb.) - Abkhazia, Adjara, Mtskheta-Mtianeti, Shida Kartli [Brotherus 1892, Abramov \& Abramova 1953a, Abramov et al. 1986, Chikovani 1986, Chikovani \& Svanidze 2004; TBI, VGBI].

\section{Aytoniaceae Cavers}

\section{Mannia Corda}

M. androgyna (L.) A. Evans (= M. dichotoma (Raddi) S.W. Arnell, Grimaldia dichotoma Raddi) - Imereti, Mtskheta-Mtianeti, Shida Kartli [Brotherus 1892, Chikovani 1986, Chikovani \& Svanidze 2004].

M. fragrans (Balb.) Frye \& L. Clark (三 Grimaldia fragrans (Balb.) Corda ex Nees) - Mtskheta-Mtianeti, Shida Kartli [Brotherus 1892, Chikovani 1986, Chikovani \& Svanidze 2004].

\section{Plagiochasma Lehm.}

P. rupestre (J.R. Forst. \& G. Forst.) Steph. - Shida Kartli [Brotherus 1892, Chikovani 1986].

\section{Reboulia Raddi}

R. hemisphaerica (L.) Raddi s.l. - all regions [Brotherus 1892, Abramova \& Abramov 1953b, Abramov et al. 1963, 1966, Chikovani 1986, Chikovani \& Svanidze 2004, Bakalin et al. 2013; VBGI.

Cleveaceae Cavers

Clevea Lindb.

C. byalina (Sommerf.) Lindb. (= Athalamia byalina (Sommerf.) S. Hatt., Clevea suecica (Lindb. ex Gottsche \& Rabenh.) Lindb.) - Mtskheta-Mtianeti [Brotherus 1892, Chikovani 1986, Chikovani \& Svanidze 2004].

Peltolepis Lindb.

P. quadrata (Saut.) Müll. Frib. - Mtskheta-Mtianeti [Chikovani 1986, Chikovani \& Svanidze 2004].

Conocephalaceae Müll. Frib. ex Grolle

Conocephalum Hill

C. conicum (L.) Dumort. s. str. (三 Fegatella conica (L.) Corda) - all regions [Brotherus 1892, Abramova \& Abramov 1953a, Abramov et al. 1963, 1964, 1966, Duda 1982, Chikovani 1986, Váňa \& Ignatov 1990, Chikovani \& Svanidze 2004, Bakalin et al. 2013; VBGI.

Ricciaceae Rchb.

Riccia L.

R. bifurca Hoffm. - Mtskheta-Mtianeti, Shida Kartli [Brotherus 1892, Chikovani 1986, Chikovani \& Svanidze 2004].

R. ciliata Hoffm. var. intumescens Bisch. - Mtskheta-Mtianeti [Chikovani 1986, Chikovani \& Svanidze 2004].

R. ciliifera Link ex Lindenb. - Mtskheta-Mtianeti [Chikovani 1986, Chikovani \& Svanidze 2004].

R. fluitans L. - Samtskhe-Javakheti, Shida Kartli [Chikovani 1986, Chikovani \& Svanidze 2004].

R. frostii Austin - Adjara [Chikovani 1986, Chikovani \& Svanidze 2004].

R. sorocarpa Bisch. - Mtskheta-Mtianeti, Samtskhe-Javakheti, Shida Kartli [Brotherus 1892, Abramova \& Abramov 1953a, Chikovani 1986, Chikovani \& Svanidze 2004; TBI, VBGI].

Pelliaceae H. Klinggr.

\section{Pellia Raddi}

P. endiviifolia (Dicks.) Dumort. (= P. calycina (Taylor) Nees, P. fabroniana s. auct. ) - all regions [Brotherus 1892, Abramova \& Abramov 1953a, Abramov et al. 1963, 1964, 1966, 1986, Chikovani 1986, Váňa \& Ignatov 1990, Chikovani \& Svanidze 2004].

P. epipbylla (L.) Corda - all regions [Brotherus 1892, Abramov et al. 1964, 1966, Duda 1982, Chikovani 1986, Chikovani \& Svanidze 2004].

P. neesiana (Gottsche) Limpr. - Adjara, Mtskheta-Mtianeti, Samtskhe-Javakheti [Abramov et al. 1964, 1966, Duda 1982, Chikovani 1986, Chikovani \& Svanidze 2004, Bakalin et al. 2013; VBGI].

Fossombroniaceae Hazsl.

\section{Fossombronia Raddi}

F. foveolata Lindb. (= F. dumortieri (Huebener \& Genth) Lindb., F. dumortieri var. robusta Lindb. ex Ladyz. ) - Guria, Samegrelo, Shida Kartli [Chikovani 1972, 1973, 1986, Chikovani \& Svanidze 2004].

F. pusilla (L.) Nees - Abkhazia [Chikovani 1986, Chikovani \& Svanidze 2004] 


\section{Pallaviciniaceae Mig.}

\section{Pallavicinia Gray}

P. lyellii (Hook.) Gray - Adjara [Abramov et al. 1966] - This record in Abramov et al. (1966) was overlooked by Chikovani \& Svanidze (2004).

Moerckiaceae K.I. Goebel ex Stotler \& Crand.-Stotl.

Moerckia Gottsche

M. flotoviana (Nees) Schiffn. - Shida Kartli [Abramova \& Abramov 1953a, Chikovani 1986, Chikovani \& Svanidze 2004] - Chikovani \& Svanidze (2004) included this taxon twice for the same region, the first time as aforementioned and the second time as "Moerckia flotoviana (Nees) Schiffn. f. flotoviana-Shida Kartli".

Metzgeriaceae H. Klinggr.

\section{Metzgeria Raddi}

$M$. conjugata Lindb. (= M. conjugata Lindb. var. epilosa Steph. ex Broth.) - all regions [Brotherus 1892, Abramova \& Abramov 1953a, Abramov et al. 1963, 1966, Váňa \& Ignatov 1990, Chikovani \& Svanidze 2004, Bakalin et al. 2013; VBG].

M. furcata (L.) Corda (= M. furcata var. ulvula Nees) - all regions [Brotherus 1892, Abramov et al. 1963, 1964, Chikovani 1986, Váňa \& Ignatov 1990, Chikovani \& Svanidze 2004, Bakalin et al. 2013; VBGI].

M. pubescens (Schrank) Raddi (= Apometzgeria pubescens (Schrank) Kuwah.) - all regions [Brotherus 1892, Abramova \& Abramov 1953a, Chikovani 1986, Chikovani \& Svanidze 2004; TBI, VBGI].

M. temperata Kuwah. - Adjara [Bakalin et al. 2013; VBG].

Aneuraceae H. Klinggr.

Aneura Dumort.

A. pinguis (L.) Dumort. (= Riccardia pinguis (L.) Gray) - Abkhazia, Adjara, Kvemo Kartli, Samtskhe-Javakheti, Shida Kartli, [Brotherus 1892, Abramov et al. 1966, Chikovani 1986, Váňa \& Ignatov 1990, Chikovani \& Svanidze 2004].

\section{Riccardia Gray}

R. chamedryfolia (With.) Grolle (= Riccardia sinuata (Hook.) Trevis., R. pinnatifida (F. Weber) Carruth., Aneura pinnatifida (F. Weber) - Samegrelo-Zemo Svaneti, Kvemo Svaneti [Chikovani 1986, Chikovani \& Svanidze 2004].

R. latifrons (Lindb.) Lindb. - all regions [Abramova \& Abramov 1953a, Abramov et al. 1964, Chikovani 1986, Chikovani \& Svanidze 2004].

R. multifida (L.) Grav - Adjara [Brotherus 1892, Abramov et al. 1966, Chikovani 1986, Chikovani \& Svanidze 2004 , Bakalin et al. 2013; VBGI.

R. palmata (Hedw.) Carruth. (= Aneura palmata (Hedw.) Dumort.) - Dusheti, Kakheti, Racha-Lechkhumi, SamtskheJavakheti, Kvemo Svaneti [Abramov et al. 1963, 1964 Chikovani 1986, Chikovani \& Svanidze 2004].

\section{Porellaceae Cavers}

\section{Porella L.}

P. arboris-vitae (With. ) Grolle (= P. laevigata (Schrad.) Pfeiff., P. laevigata var. thuja (Hook.) Arnell, P. caucasica Steph. ?) - all regions [Brotherus 1892, Abramov et al. 1963, Chikovani 1986, Chikovani \& Svanidze 2004]. Porella caucasica was regarded as synonym of $P$. laevigata in Chikovani \& Svanidze (2004), but they did not provide nomenclatural citation (therefore synonymy is invalid) and we do not know whether this synonymyzation was based on study of types or only description.
P. cordaeana (Huebener) Moore (= Madotheca rivularis Nees) - Abkhazia, Kakheti, Racha-Lechkhumi, Kvemo Kartli, Samtskhe-Javakheti, Shida Kartli [Brotherus 1892, Abramov et al. 1963, Chikovani 1986, Chikovani \& Svanidze 2004; TBI, VBGI]

P. platyphylla (L.) Pfeiff. (三 Madotheca platyphylla (L.) Dumort.) - all regions [Brotherus 1892, Abramova \& Abramov 1953a, Abramov et al. 1963, 1964, Chikovani 1986, Váňa \& Ignatov 1990, Chikovani \& Svanidze 2004, Bakalin et al. 2013; VBGI.

P. platyphylloidea (Schwein.) Lindb. (三 Madotheca platyphylloidea (Schwein.) Nees) - Racha-Lechkumi [Brotherus 1892, Chikovani 1986, Chikovani \& Svanidze 2004]. Chikovani \& Svanidze (2004) regarded this name as synonymous with P. baueri (Schiffn.) C. Jens.; the reasons for this are unclear to us.

Radulaceae Müll. Frib.

Radula Dumort.

R. complanata (L.) Dumort. (= R. complanata (L.) Dumort. var. alpestris (Lindb. ex Berggr.) Lindb., R. complanata (L.) Dumort. var. cucullifolia Steph. ex Broth.) - all regions [Brotherus 1892, Abramova \& Abramov 1953a, Abramov et al. 1963, 1966, Duda 1982, Chikovani 1986, Chikovani \& Svanidze 2004, Bakalin et al. 2013; VBGI].

R. lindenbergiana Gottsche ex C. Hartm. (= Radula commutata Gottsche ex Limpr.) - all regions [Brotherus 1892, Abramova \& Abramov 1953a, Abramov et al. 1963, 1966, Duda 1982, Chikovani 1986, Váňa \& Ignatov 1990, Chikovani \& Svanidze 2004, Bakalin et al. 2013; VBGI].

Jubulaceae H. Klinggr.

Jubula Dumort.

J. hutchinsiae subsp. caucasica Konstant. \& Vilnet - Adjara, Imereti, Shida Kartli - Jubula butchinsiae s.l. was overlooked by compilers of the checklist of Georgian liverworts (Chikovani \& Svanidze 2004), although it was previously recorded for Georgia at least three times. We suggest that records under the names Jubula hutchinsiae subsp. javanica (Steph.) Verd. (Abramova \& Abramov 1953a, Abramov et al. 1966) and Frullania butchinsiae (Hook.) Nees var. integrifolia (Nees) Lindb. (Brotherus 1892) should belong to this taxon. Recently the subsp. caucasica was also recorded for Georgia by Kürschner et al. (2013) [VBGI]

\section{Frullaniaceae Lorch}

Frullania Raddi

F. dilatata (L.) Dumort. ( = F. dilatata (L.) Dumort. var. anomala Corb.) - Imereti, Kakheti, Kvemo Kartli, Racha-Lechkhumi, Samtskhe-Javakheti, Shida Kartli [Abramova \& Abramov 1953a, Abramov et al. 1963, 1964, 1966, Duda 1982, Chikovani 1986, Váňa \& Ignatov 1990, Chikovani \& Svanidze 2004, Bakalin et al. 2013; VBGI.

F. fragilifolia (Taylor) Gottsche, Lindenb. \& Nees - MtskhetaMtianeti [Chikovani 1986, Chikovani \& Svanidze 2004].

F. jackii Gottsche - Adjara [Bakalin et al. 2013; VBGI].

F. tamarisci (L.) Dumort. - all regions [Brotherus 1892, Abramova \& Abramov 1953a, Abramov et al. 1964, Duda 1982, Chikovani 1986, Chikovani \& Svanidze 2004, Bakalin et al. 2013; VBGI]

F. parvistipula Steph. (= F. tenera Lindb. ex Steph., nom. illeg., non Spruce) - Kvemo Kartli, Samtskhe-Javakheti, Shida Kartli [Brotherus 1892].

Lejeuneaceae Cavers

Cololejeunea (Spruce) Steph.

C. calcarea (Lib.) Steph. (= Lejeunea calcarea Lib.) - Abkha- 
zia, Adjara, Imereti Brotherus 1892, Duda 1982, Chikovani 1986, Váňa \& Ignatov 1990, Chikovani \& Svanidze 2004, Bakalin et al. 2013; VBGI].

C. rossettiana (C. Massal.) Schiffn. - Abkhazia, Shida Kartli [Chikovani 1986, Chikovani \& Svanidze 2004].

\section{Lejeunea Lib.}

L. cavifolia (Ehrh.) Lindb. - all regions [Brotherus 1892, Abramova \& Abramov 1953a, Abramov et al. 1963, 1966 Duda 1982, Chikovani 1986, Váňa \& Ignatov 1990 Chikovani \& Svanidze 2004, Bakalin et al. 2013; VBGI].

L. patens Lindb. - Abkhazia, Adjara, Guria, Shida Kartli [Brotherus 1892, Chikovani 1986, Chikovani \& Svanidze 2004].

\section{Ptilidiaceae H. Klinggr.}

\section{Ptilidium Nees}

P. ciliare (L.) Hampe - all regions [Brotherus 1892, Chikovani 1986, Chikovani \& Svanidze 2004].

P. pulcherrimum (Weber) Vain. - Guria, Kvemo Kartli, Samtskhe-Javakheti, Shida Kartli, Kvemo Svaneti [Brotherus 1892, Abramova \& Abramov 1953a, Abramov et al. 1964, Duda 1982, Chikovani 1986, Chikovani \& Svanidze 2004].

\section{Pseudolepicoleaceae Fulford \& J. Taylor}

Blepharostoma (Dumort.) Dumort.

B. trichophyllum (L.) Dumort. (三 Jungermannia trichophylla L.) all regions [Brotherus 1892, Abramova \& Abramov 1953a, Abramov et al. 1964, 1986, Duda 1982, Chikovani 1986, Chikovani \& Svanidze 2004, Bakalin et al. 2013; VBGI].

\section{Trichocoleaceae Nakai}

Trichocolea Dumort.

T. tomentella (Ehrh.) Dumort. - Abkhazia, Adjara [Chikovani 1986, Chikovani \& Svanidze 2004].

\section{Lepidoziaceae Limpr.}

\section{Bazzania Gray}

B. flaccida (Dumort.) Grolle (= B. denudata auct.) - Adjara [Abramov et al. 1966, Chikovani 1986, Chikovani \& Svanidze 2004].

B. tricrenata (Wahlenb.) Lindb. (= Mastigobryum deflexum (Mart.) Nees) - Adjara, Samtskhe-Javakheti, Shida Kartli, Kvemo Svaneti [Brotherus 1892, Abramov et al. 1964, 1966, Chikovani 1986, Chikovani \& Svanidze 2004; TBI, VBGI.

B. trilobata (L.) Gray - Abkhazia, Adjara, Samtskhe-Javakheti [Abramov et al. 1964, Chikovani 1986, Chikovani \& Svanidze 2004, Bakalin et al. 2013; VBGI].

\section{Lepidozia (Dumort. ) Dumort.}

L. reptans (L.) Dumort. - all regions [Brotherus 1892, Abramova \& Abramov 1953a, Abramov et al. 1963, 1964, 1966 Chikovani 1986, Chikovani \& Svanidze 2004; TBI, VBGI].

Lophocoleaceae Vanden Berghen

Chiloscyphus Corda

C. fragilis (A. Roth) Schiffn. - Adjara [Bakalin et al. 2013; VBGI.

C. pallescens (Ehrh. ex Hoffm.) Dumort. - all regions [Brotherus 1892, Abramova \& Abramov 1953a, Abramov et al. 1963, 1964, 1966, Chikovani 1986, Chikovani \& Svanidze 2004].

C. polyanthos (L.) Corda - all regions [Brotherus 1892, Abramov et al. 1963, 1964, 1966, Chikovani 1986, Chikovani \& Svanidze 2004].
Lophocolea (Dumort.) Dumort.

L. bidentata (L.) Dumort. - Abkhazia, Adjara, Kvemo Svaneti [Abramov et al. 1966, Chikovani 1986, Chikovani \& Svanidze 2004, Bakalin et al. 2013; VBGI].

L. cuspidata (Nees) Limpr. - all regions [Abramov et al. 1966, Chikovani 1986, Chikovani \& Svanidze 2004]. L. cuspidata is usually regarded as a synonym of $L$. bidentata, but the name has frequently been misapplied and it may be that what here is referred to as $L$. cuspidata really is $L$. coadunata (Sw.) Mont.

L. heterophylla (Schrad.) Dumort. - Abkhazia, Adjara, Kakheti, Kvemo Svaneti, Samtskhe-Javakheti Brotherus 1892, Abramova \& Abramov 1953a, Abramov et al. 1963, 1964, 1966, Abramov et al. 1986, Duda 1982, Chikovani 1986, Váňa \& Ignatov 1990, Chikovani \& Svanidze 2004, Bakalin et al. 2013; VBGI].

L. minor Nees - all regions [Brotherus 1892, Abramova \& Abramov 1953a, Abramov et al. 1964, 1966, 1986, Chikovani 1986, Chikovani \& Svanidze 2004; VBGI].

Plagiochilaceae Müll. Frib.

Plagiochila (Dumort.) Dumort.

P. asplenioides (L.) Dumort. (= P. major (Nees) S.W. Arnell) all regions [Brotherus 1892, Abramova \& Abramov 1953a, Abramov et al. 1963, 1966, 1986, Chikovani 1986, Chikovani \& Svanidze 2004]. Most probably the majority of records of this taxon for Georgia belong to $P$. porelloides, although Plagiochila asplenioides s. str. should also be more or less common there.

P. porelloides (Torr. ex Nees) Lindenb. - Abkhazia, Adjara [Abramov et al. 1986, Váňa \& Ignatov 1990, Bakalin et al. 2013; VBGI.

Pedinophyllum Lindb.

P. interruptum (Nees) Kaal. (三 Leptoscyphus interruptus (Nees) Lindb.) - Abkhazia, Imereti, Racha-Lechkumi [Brotherus 1892, Chikovani 1986, Váňa \& Ignatov 1990, Chikovani \& Svanidze 2004]

Adelanthaceae Grolle

Syzygiella Spruce

S. autumnalis (DC.) K. Feldberg, Váňa, Hentschel \& Heinrichs (三 Jamesoniella autumnalis (DC.) Steph., Jungermannia autumnalis DC., J. schraderi Mart.) - all regions [Brotherus 1892, Abramova \& Abramov 1953a, Abramov et al. 1963, 1966, Chikovani 1986, Chikovani \& Svanidze 2004, Bakalin et al. 2013; VBGI].

\section{Cephaloziaceae Mig.}

Cephalozia (Dumort.) Dumort.

C. bicuspidata (L.) Dumort. - all regions [Brotherus 1892, Abramov et al. 1966, Chikovani 1986, Chikovani \& Svanidze 2004; VBGI. Chikovani \& Svanidze (2004) also recorded as a separate species C. lammersiana (Huebener) Carrington for Adjara, but we follow Konstantinova et al. (2009), and most of the other recent literature referring the latter name to the synonymy of C. bicuspidata.

C. lacinulata (J.B. Jack ex Gottsche \& Rabenh.) Spruce - Adjara, Kvemo Svaneti [Chikovani \& Svanidze 2004, Bakalin et al. 2013; VBGI].

\section{Fuscocephaloziopsis Fulford}

F. catenulata (Huebener) Váňa \& L. Söderstr. - Adjara, Mtskheta-Mtianeti, Shida Kartli [Abramov et al. 1966, Chikovani 1986, Chikovani \& Svanidze 2004, Bakalin et al. 2013; VBGI].

F. connivens (Dicks.) Váňa \& L. Söderstr. (三 Jungermannia con- 
nivens Dicks.) - all regions [Brotherus 1892, Abramova \& Abramov 1953a, Chikovani 1986, Chikovani \& Svanidze 2004].

F. leucantha (Spruce) Váňa \& L. Söderstr. - Kvemo Svaneti [Chikovani 1986, Chikovani \& Svanidze 2004].

F. lunulifolia (Dumort.) Váňa \& L. Söderstr. (= C. media Lindb.) - Adjara, Samtskhe-Javakheti, Kvemo Kartli [Abramov et al. 1966, Chikovani \& Svanidze 2004].

F. pleniceps (Austin) Váňa \& L. Söderstr. - Shida Kartli [Chikovani 1986, Chikovani \& Svanidze 2004].

\section{Nowellia Mitt.}

N. curvifolia (Dicks.) Mitt. (三 Cephalozia curvifolia (Dicks.) Dumort.) - Abkhazia, Adjara, Kakheti, Kvemo Kartli, Racha-Lechkhumi Brotherus 1892, Abramov et al. 1963 , Duda 1982, Chikovani 1986, Váňa \& Ignatov 1990, Chikovani \& Svanidze 2004, Bakalin et al. 2013; VBGI.

Odontoschismataceae Schljakov (= Cephaloziaceae subfam. Odontoschismatoideae H. Buch ex Grolle)

Odontoschisma (Dumort.) Dumort.

O. denudatum (Mart.) Dumort. - Kakheti [Abramov et al. 1963, Duda 1982, Chikovani 1986, Chikovani \& Svanidze 2004].

O. fluitans (Nees) L. Söderstr. \& Váňa - Mtskheta-Mtianeti [Chikovani 1972, 1986, Chikovani \& Svanidze 2004].

\section{Cephaloziellaceae Douin}

Cephaloziella (Spruce) Schiffn.

C. divaricata $(\mathrm{Sm}$.) Schiffn. (= C. divaricata $(\mathrm{Sm}$.) Schiffn. var. scabra (M. Howe) Haynes, C. starkei auct.) - all regions Brotherus 1892, Abramov et al. 1963, 1966, Chikovani 1986, Chikovani \& Svanidze 2004, Bakalin et al. 2013; VBGI, TBI].

C. hampeana (Nees) Schiffn. ex Loeske - Adjara [Chikovani 1986, Chikovani \& Svanidze 2004].

C. rubella (Nees) Warnst. - Adjara, Mtskheta-Mtianeti [Chikovani 1972, 1986, Chikovani \& Svanidze 2004].

C. turneri (Hook. ) Müll. Frib. - Abkhazia, Adjara [Chikovani 1973, 1986, Chikovani \& Svanidze 2004].

Anastrophyllaceae L. Söderstr., De Roo \& Hedd.

Anastrophyllum (Spruce) Steph.

A. michauxii (F. Weber) H. Buch - Racha-Lechkhumi [Chikovani 1986, Chikovani \& Svanidze 2004].

\section{Barbilophozia Loeske}

B. barbata (Schmidel ex Schreb.) Loeske - all regions [Brotherus 1892, Abramov et al. 1963, 1986, Chikovani 1986, Chikovani \& Svanidze 2004; TBI, VBGI].

B. hatcheri (A. Evans) Loeske - all regions [Abramova \& Abramov 1953a, Abramov et al. 1963, 1964, Chikovani 1986, Chikovani \& Svanidze 2004, Bakalin et al. 2013; VBGI].

B. lycopodioides (Wallr.) Loeske - all regions [Brotherus 1892 Abramova \& Abramov 1953a, Abramov et al. 1963, 1986, Chikovani 1986, Chikovani \& Svanidze 2004].

Gymnocolea (Dumort. ) Dumort.

G. inflata (Huds.) Dumort. (三 Jungermannia inflata Huds.) Imereti [Brotherus 1892, Chikovani 1986, Chikovani \& Svanidze 2004]

Isopaches $\mathrm{H}$. Buch

I. bicrenatus (Schmidel ex Hoffm.) H. Buch (三 Lophozia bicrenata (Schmidel ex Hoffm.) Dumort. - Mtskheta-Mtianeti, Kvemo Svaneti, Kvemo Kartli [Chikovani 1986, Chikovani \& Svanidze 2004]
Neoorthocaulis L. Söderstr., De Roo \& Hedd.

N. attenuatus (Mart.) L. Söderstr., De Roo \& Hedd. (= Barbilophozia gracilis (Schleich. ex DC. ) Müll. Frib.) - Abkhazia, Kvemo Svaneti [Chikovani 1986, Chikovani \& Svanidze 2004].

N. floerkei (F. Weber \& D. Mohr) L. Söderstr., De Roo \& Hedd. (三 Barbilophozia floerkei (F. Weber \& D. Mohr) Loeske, Lophozia floerkei (F. Weber \& D. Mohr) Schiffn., Jungermannia lycopodioides Wallr. var. floerkei (F. Weber \& D. Mohr) Lindb.) - Mtskheta-Mtianeti, Shida Kartli, Samtskhe-Javakheti [Brotherus 1892, Abramova \& Abramov 1953a, Chikovani 1986, Chikovani \& Svanidze 2004].

Pseudolophozia Konstant. \& Vilnet

Pseudolophozia sudetica (Nees ex Huebener) Konstant. \& Vilnet (三 Lophozia sudetica (Nees ex Huebener) Grolle, L. alpestris auct.) - all regions [Chikovani 1972, 1986, Chikovani \& Svanidze 2004; TBI, VBGI]. This species was confirmed for Kakheti only; see discussion on this taxon in Bakalin \& Tigishvili (2013).

Schljakovianthus Konstant. \& Vilnet

S. quadrilobus (Lindb.) Konstant. \& Vilnet (三 Lophozia quadriloba (Lindb.) A. Evans) - Shida Kartli [Abramova \& Abramov 1953a, Chikovani 1986, Chikovani \& Svanidze 2004].

Sphenolobus (Lindb.) Berggr.

Sphenolobus minutus (Schreb. ex D. Crantz) Berggr. (三 Jungermannia minuta Schreb. ex D. Crantz) - all regions [Abramova \& Abramov 1953a, Abramov et al. 1966, Chikovani 1986, Chikovani \& Svanidze 2004, Bakalin et al. 2013; VBGI].

Scapaniaceae Mig.

Diplophyllum (Dumort.) Dumort.

D. albicans (L.) Dumort. - Abkhazia, Adjara, Imereti, Kakheti, Kvemo Svaneti [Brotherus 1892, Abramov et al. 1963, 1966, Duda 1982, Chikovani 1986, Chikovani \& Svanidze 2004, Bakalin et al. 2013; VBGI].

D. obtusifolium (Hook.) Dumort. - Mtskheta-Mtianeti, Kvemo Svaneti [Chikovani 1986, Chikovani \& Svanidze 2004].

D. taxifolium (Wahlenb.) Dumort. - Abkhazia, Guria, Kvemo Svaneti, Mtskheta-Mtianeti, Shida Kartli Brotherus 1892, Abramova \& Abramov 1953a, Abramov et al. 1966, 1986, Chikovani 1986, Chikovani \& Svanidze 2004].

Lophozia (Dumort.) Dumort.

L. ascendens (Warnst.) R.M. Schust. - Adjara, Kvemo Kartli, Samtskhe-Javakheti [Abramov et al. 1964, 1966, Chikovani 1986, Chikovani \& Svanidze 2004].

L. lantratoviae Bakalin - Mtskheta-Myianeti [Bakalin \& Tigishvili 2013; TBI, VBGI].

L. guttulata (Lindb. \& Arnell) A. Evans - Adjara, Guria, Kvemo Kartli, Sakachavo [Duda 1982, Bakalin \& Tigishvili 2013; TBI, VBGI].

L. silvicola $\mathrm{H}$. Buch (= L. porphyrolenca (Nees) Schiffn.) all regions [Abramov et al. 1966, Chikovani \& Svanidze 2004]. Lophozia porphyroleuca is sometimes regarded as a synonym of $L$. longiflora or $L$. guttulata, although Schljakov (1981), based on a study of type specimens, showed this name is a synonym of $L$. ventricosa sensu Schljakov (i.e. $L$. silvicola in the current view).

L. ventricosa (Dicks.) Dumort. (= Jungermannia porphyroleuca auct., J. ventricosa Dicks., ?J. porphyroleuca Nees var. flavidula Lindb.) - all regions [Brotherus 1892, Abramova \& Abramov 1953a, Abramov et al. 1964, 1966, Duda 1982, Chikovani 1986, Chikovani \& Svanidze 2004, Bakalin \& Tigishvili, 2013].

L. wenzelii (Nees) Steph. - all regions [Chikovani 1986, Chi- 
kovani \& Svanidze 2004]. Most probably these records belong to var. massularioides Bakalin of the species.

L. wenzelii (Nees) Steph. var. massularioides Bakalin - Adjara [Bakalin \& Tigishvili 2013; TBI, VBGI].

Lophoziopsis Konstant. \& Vilnet

L. excisa (Dicks. ) Konstant. \& Vilnet (三 Lophozia excisa (Dicks.) Dumort.) - all regions [Abramov et al. 1964, Chikovani 1986, Chikovani \& Svanidze 2004, Bakalin \& Tigishvili 2013; VBGI.

L. longidens (Lindb.) Konstant. \& Vilnet - Kakheti [Bakalin \& Tigishvili 2013; TBI, VBGI]

Obtusifolium S.W. Arnell

O. obtusum (Lindb.) S.W. Arnell (三 Barbilophozia obtusa (Lindb.) H. Buch - Kakheti [Abramov et al. 1963, Chikovani 1986, Chikovani \& Svanidze 2004].

Oleolophozia L. Söderstr., De Roo \& Hedd.

O. perssonii (H. Buch \& S.W. Arnell) L. Söderstr., De Roo \& Hedd. - Kvemo Svaneti [Bakalin \& Tigishvili 2013; TBI, VBGI].

Scapania (Dumort. ) Dumort.

S. aequiloba (Schwägr.) Dumort. - Adjara, Imereti, Kvemo Svaneti, Mtskheta-Mtianeti, Racha-Lechkhumi, Shida Kartli [Brotherus 1892, Abramov et al. 1966, Chikovani 1986, Chikovani \& Svanidze 2004].

S. apiculata Spruce - Mtskheta-Mtianeti [Chikovani 1986, Chikovani \& Svanidze 2004].

S. aspera M. Bernet \& Bernet (三 Martinellius asperus (M. Bernet \& Bernet) Broth.) - Abkhazia, Kvemo Svaneti, Mtskheta-Mtianeti, Shida Kartli [Brotherus 1892, Chikovani 1986, Chikovani \& Svanidze 2004].

S. calcicola (Arnell \& J. Perss. ) Ingham - Abkhazia, Kvemo Svaneti [Chikovani 1986, Chikovani \& Svanidze 2004].

S. cuspiduligera (Nees) Müll. Frib. - Mtskheta-Mtianeti [Chikovani 1986, Chikovani \& Svanidze 2004].

S. helvetica Gottsche - Kakheti [Abramov et al. 1963, Chikovani 1986, Chikovani \& Svanidze 2004].

S. irrigua (Nees) Nees (三 Martinellius irriguus (Nees) Lindb.) - all regions [Brotherus 1892, Abramov et al. 1963, Chikovani 1986, Chikovani \& Svanidze 2004].

S. nemorea (L.) Grolle - all regions [Brotherus 1892, Abramov et al. 1963, 1966, Duda 1982, Chikovani 1986, Chikovani \& Svanidze 2004, Bakalin et al. 2013; VBGI].

S. mucronata H. Buch - Shida Kartli [Abramov et al. 1964, Chikovani 1986, Chikovani \& Svanidze 2004].

S. subalpina (Nees ex Lindenb. ) Dumort. (三 Martinellius subalpinus (Nees ex Lindenb. ) Trevis. ) - Mtskheta-Mtianeti, Shida Kartli [Brotherus 1892, Chikovani 1986, Chikovani \& Svanidze 2004].

S. uliginosa (Sw. ex Lindenb. ) Dumort. - Adjara, SamtskheJavakheti [Chikovani 1973, 1986, Chikovani \& Svanidze 2004].

S. undulata (L.) Dumort. (= S. undulata (L.) Dumort. f. dentata (Dumort.) Jørg., Martinellius resupinatus (L.) Gray) - all regions [Brotherus 1892, Abramov et al. 1963, 1966, Duda 1982, Chikovani 1986, Chikovani \& Svanidze 2004, Bakalin et al. 2013; VBGI].

S. verrucosa Heeg - Adjara, Kakheti, Mtskheta-Mtianeti [Abramov et al. 1963, 1966, Chikovani 1986, Chikovani \& Svanidze 2004, Bakalin et al. 2013; VBGI].

Schistochilopsis (N. Kitag.) Konstant.

S. incisa (Schrad.) Konstant. (三 Lophozia incisa (Schrad.) Dumort., Jungermannia incisa Schrad.) - all regions [Brotherus
1892, Abramova \& Abramov 1953a, Abramov et al. 1966, Duda 1982, Chikovani 1986, Chikovani \& Svanidze 2004].

Trilophozia (R.M. Schust.) Bakalin

T. quinquedentata (Huds.) Bakalin - all regions [Brotherus 1892, Abramova \& Abramov 1953a, Abramov et al. 1963, Chikovani 1986, Chikovani \& Svanidze 2004; TBI, VBGI].

Tritomaria Schiffn. ex Loeske

T. exsecta (Schmidel ex Schrad. ) Schiffn. ex Loeske (三 Sphenolobus exsectus (Schmidel ex Schrad.) Steph., Jungermannia exsecta Schmidel ex Schrad.) - all regions [Brotherus 1892, Abramova \& Abramov 1953a, Abramov et al. 1963, 1966, Chikovani 1986, Chikovani \& Svanidze 2004; TBI, VBGI].

T. exsectiformis (Breidl.) Schiffn. ex Loeske - Kvemo Kartli, Samtskhe-Javakheti, Shida Kartli [Chikovani 1986, Chikovani \& Svanidze 2004].

Myliaceae Schljakov

Mylia Gray

M. taylorii (Hook.) Gray - Adjara [Chikovani \& Svanidze 2004].

Arnelliaceae Nakai

Southbya Spruce

S. tophacea (Spruce) Spruce (= S. stillicidiorum (Raddi) Lindb.) - Abkhazia, Kvemo Kartli, Samtskhe-Javakheti, Shida Kartli [Chikovani 1986, Váňa \& Ignatov 1990, Chikovani \& Svanidze 2004]

Calypogeiaceae Arnell

Calypogeia Raddi

C. arguta Nees \& Mont. - Adjara, Samegrelo [Abramov et al. 1966, Chikovani 1973, Duda 1982, Chikovani 1986, Chikovani \& Svanidze 2004, Bakalin et al. 2013; VBGI.

C. azurea Stotler \& Crotz (= C. trichomanis (L.) Corda) - all regions [Brotherus 1892, Abramova \& Abramov 1953a, Abramov et al. 1966, 1986, Chikovani 1986, Chikovani \& Svanidze 2004]. This name in Chikovani \& Svanidze (2004) probably reflects many old records that regard most Calypogeia species as C. trichomanis. The real distribution of C. aqurea in Georgia remains ambiguous.

C. fissa (L.) Raddi-Abkhazia, Adjara, Samegrelo [Chikovani 1973, 1986, Duda 1982, Chikovani \& Svanidze 2004, Bakalin et al. 2013; VBGI].

C. muelleriana (Schiffn.) Müll. Frib. - Abkhazia, Adjara, Samegrelo [Abramov et al. 1966, Chikovani 1986, Chikovani \& Svanidze 2004; TBI, VBGI].

C. neesiana (C. Massal. \& Carestia) Müll. Frib. - Kakheti, Mtskheta-Mtianeti, Shida Kartli, Kvemo Kartli [Abramova \& Abramov 1953a, Chikovani 1973, 1986, Chikovani \& Svanidze 2004, Abramov et al. 1986].

C. sphagnicola (Arnell \& J. Perss.) Warnst. \& Loeske - Mtskheta-Mtianeti, Kvemo Svaneti [Chikovani 1973, 1986, Chikovani \& Svanidze 2004].

C. suecica (Arnell \& J. Perss.) Müll. Frib. - Abkhazia, Kvemo Kartli [Chikovani 1973, 1986, Chikovani \& Svanidze 2004; TBI, VBGI].

\section{Delavayellaceae R.M. Schust.}

\section{Liochlaena Nees}

L. lanceolata Nees (三 Jungermannia lanceolata sensu Schrad.) - all regions [Brotherus 1892, Abramova \& Abramov 1953b, Abramov et al. 1963, 1966, Chikovani 1986, Chikovani \& Svanidze 2004].

L. subulata (A. Evans) Schljakov - Adjara [Bakalin et al. 2013; VBGI]. 


\section{Jungermanniaceae Rchb.}

\section{Jungermannia $\mathrm{L}$.}

J. atrovirens Dumort. (三 Haplozia atrovirens (Dumort.) Dumort., = Jungermannia tristis Nees, Haplozia tristis (Nees) Dumort., Jungermannia riparia Taylor) - Abkhazia, Mtskheta-Mtianeti, Racha-Lechkumi, Shida Kartli [Brotherus 1892, Duda 1982, Chikovani 1986, Chikovani \& Svanidze 2004, Bakalin et al. 2013; VBGI. Chikovani \& Svanidze (2004) listed this species twice, firstly as Haplozia atrovirens and secondly as $H$. tristis.

J. exsertifolia Steph. subsp. cordifolia (Dumort.) Váňa (三J. cordifolia Hook., nom. illeg., non Brot., Haplozia cordifolia Dumort., Aplozia cordifolia Dumort. ) - Adjara, Kvemo Svaneti, Mtskheta-Mtianeti, Racha-Lechkumi [Brotherus 1892, Abramova \& Abramov 1953b, Chikovani 1986, Chikovani \& Svanidze 2004].

\section{Mesoptychia (Lindb.) A. Evans}

M. badensis (Gottsche ex Rabenh.) L. Söderstr. \& Váňa (三 Lophoria badensis (Gottsche ex Rabenh.) Schiffn. ) - Mtskheta-Mtianeti, Shida Kartli [Abramova \& Abramov 1953a, Chikovani 1986, Chikovani \& Svanidze 2004].

M. bantriensis (Hook.) L. Söderstr. \& Váňa (= Lophozia hornschuchiana (Nees) Macoun) - Adjara, Kakheti, Shida Kartli, Kvemo Svaneti [Abramova \& Abramov 1953a, Abramov et al. 1963, Chikovani 1986, Chikovani \& Svanidze 2004].

M. collaris (Nees) L. Söderstr. \& Váňa (= Lophozia muelleri (Nees ex Lindenb.) Dumort., Jungermannia muelleri Nees ex Lindenb.) - all regions [Brotherus 1892, Abramova \& Abramov 1953a, Abramov et al. 1963, Chikovani 1986, Chikovani \& Svanidze 2004].

M. heterocolpos (Thed. ex Hartm.) L. Söderstr. \& Váňa (三 Lophozia heterocolpos (Thed. ex Hartm.) M. Howe) - Kakheti, Shida Kartli [Abramova \& Abramov 1953a, Abramov et al. 1963, Chikovani 1986, Chikovani \& Svanidze 2004].

M. turbinata (Raddi) L. Söderstr. \& Váňa (三 Jungermannia turbinata Raddi, Lophozia turbinata (Raddi) Steph.) - Adjara, Imereti, Racha-Lechkumi Brotherus 1892, Chikovani 1986, Chikovani \& Svanidze 2004].

\section{Endogemmataceae Konstant., Vilnet \& A.V. Troitsky}

Endogemma Konstant., Vilnet \& A. V. Troitsky

E. caespiticia (Lindenb.) Konstant., Vilnet \& A.V. Troitsky (= Solenostoma caespiticium (Lindenb.) Steph.) - Adjara [Chikovani 1986, Chikovani \& Svanidze 2004].

Solenostomataceae Stotler \& Crand.-Stotl.

\section{Solenostoma Mitt.}

S. caucasicum (Váňa) Konstant. (三 Jungermannia caucasica Váňa) - Adjara [Váňa 1970, Duda 1982, Chikovani 1986, Chikovani \& Svanidze 2004, Bakalin et al. 2013; VBGI].

S. gracillimum (Sm.) R.M. Schust. f. crenulatum (Mitt.) Bakalin (三 Solenostoma crenulatum Mitt., Nardia crenulata (Mitt.) Lindb., = Jungermannia crenulata Sm., nom. illeg., non Schmidel, J. genthiana Huebener) - all regions [Brotherus 1892, Duda 1982, Chikovani 1986, Chikovani \& Svanidze 2004].

S. gracillimum (Sm.) R.M. Schust. f. gracillimum (三 Solenostoma crenulatum Mitt. f. gracillimum (Sm.) Vanden Berghen, Aplozia crenulata (Mitt.) Dumort. f. gracillima (Sm.) CasaresGil) - Adjara, Kvemo Kartli, Shida Kartli [Abramova \& Abramov 1953a, Chikovani \& Svanidze 2004; TBI, VBGI].

S. handelii (Schiffn.) Müll. Frib. - Adjara [Chikovani 1986, Chikovani \& Svanidze 2004].

S. levieri (Steph.) Steph. (= S. sphaerocarpum auct., $\equiv$ Nardia levieri Steph.) - Adjara, Mtskheta-Mtianeti [Brotherus 1892, Chikovani 1986, Chikovani \& Svanidze 2004, Bakalin 2014b]. See explanation on this species in Bakalin (2014b).

S. sphaerocarpum (Hook.) Steph. (三 Jungermannia sphaerocarpa Hook.) - Adjara, Shida Kartli [Brotherus 1892, Abramova \& Abramov 1953a, Abramov et al. 1966, Chikovani 1986, Chikovani \& Svanidze 2004; TBI, VBGI].

Plectocolea (Mitt. ) Mitt.

P. obovata (Nees) Lindb. - Adjara, Abkhazia, Kakheti, Kvemo Svaneti, Mtskheta-Mtianeti [Abramov et al. 1963, Chikovani 1986, Chikovani \& Svanidze 2004].

P. byalina (Lyell ex Hook.) Mitt. (三 Jungermannia byalina Lyell ex Hook.) - all regions [Abramov et al. 1966, Duda 1982, Chikovani 1986, Chikovani \& Svanidze 2004, Bakalin et al. 2013; VBGI.

P. infusca Mitt. var. memiadzei Bakalin - Adjara [Bakalin et al. 2013; VBGI].

\section{Geocalycaceae H. Klinggr.}

\section{Geocalyx Nees}

G. graveolens (Schrad.) Nees - Kvemo Kartli, Samtskhe-Javakheti [Chikovani 1986, Chikovani \& Svanidze 2004].

\section{Harpanthus Nees}

H. flotovianus (Nees) Nees - Mtskheta-Mtianeti [Chikovani 1972, 1986, Chikovani \& Svanidze 2004].

H. scutatus (F. Weber \& D. Mohr) Spruce - Abkhazia, Mtskheta-Mtianeti [Brotherus 1892, Chikovani 1986, Chikovani \& Svanidze 2004].

Antheliaceae R.M. Schust.

Anthelia (Dumort.) Dumort.

A. julacea (L.) Dumort. - Abkhazia, Mtskheta-Mtianeti, Shida Kartli [Brotherus 1892, Chikovani 1972, 1986, Chikovani \& Svanidze 2004].

A. juratzkana (Limpr.) Trevis. - Shida Kartli [Abramova \& Abramov 1953a, Chikovani 1986, Chikovani \& Svanidze 2004].

\section{Gymnomitriaceae H. Klinggr.}

Gymnomitrion Corda

G. concinnatum (Lightf.) Corda (三 Cesius concinnatus (Lightf.) Gray) - Adjara, Mtskheta-Mtianeti, Samegrelo, Shida Kartli [Brotherus 1892, Abramova \& Abramov 1953a, Abramov et al. 1966, Chikovani 1986, Chikovani \& Svanidze 2004].

G. corallioides Nees (三 Cesius coralloides (Nees) Carruth.) - Adjara, Mtskheta-Mtianeti, Shida Kartli [Brotherus 1892, Abramova \& Abramov 1953a, Chikovani 1986, Chikovani \& Svanidze 2004].

G. obtusum Lindb. (= Cesius obtusus (Lindb.) Lindb.) Mtskheta-Mtianeti [Brotherus 1892, Chikovani 1986, Chikovani \& Svanidze 2004].

\section{Marsupella Dumort.}

M. emarginata (Ehrh.) Dumort. (= Sarcocyphos ebrhardtii Corda) - Imereti, Mtskheta-Mtianeti [Brotherus 1892, Chikovani 1986, Chikovani \& Svanidze 2004, Bakalin et al. 2013; VBGI].

M. funckii (F. Weber \& D. Mohr) Dumort. (三 Sarcocyphos funckii (F. Weber \& D. Mohr) Nees, Marsupella badensis Schiffn.) - Adjara, Imereti, Kakheti, Mtskheta-Mtianeti, Racha-Lechkhumi [Brotherus 1892, Abramov et al. 1963, 1966, Chikovani 1972, 1986, Chikovani \& Svanidze 2004, Bakalin et al. 2013; VBGI].

M. sphacelata (Giesecke ex Lindenb.) Dumort. (三 Sarcocyphos sphacelatus (Giesecke ex Lindenb.) Nees) - Abkhazia, Imereti, Mtskheta-Mtianeti [Brotherus 1892, Chikovani 1986, Chikovani \& Svanidze 2004]. 
M. apiculata Schiffn. (三 Gymnomitrion apiculatum (Schiffn.) Müll. Frib.) - Adjara [Chikovani 1986, Chikovani \& Svanidze 2004]

\section{Nardia Gray}

N. compressa (Hook.) Gray - Kvemo Svaneti [Brotherus 1892 Chikovani 1986, Chikovani \& Svanidze 2004].

N. geoscyphus (De Not.) Lindb. - Mtskheta-Mtianeti, Shida Kartli [Chikovani 1986, Chikovani \& Svanidze 2004].

N. scalaris Gray - Adjara, Mtskheta-Mtianeti [Abramova \& Abramov 1953b; Chikovani 1986; Chikovani \& Svanidze 2004].

\section{INTRODUCED AND PROBABLY CURRENTLY DISAPPEARED TAXON}

Nardia assamica (Mitt.) Amakawa - Adjara [Váňa 1976]. This species was collected in Batumi Botanical Garden in the East-Asian sector of the garden and most probably was introduced from East Asia with transplanted vascular plants. Despite a special search, it was not found by Bakalin in 2013 and is probably now disappeared in the hepatic flora of the Caucasus.

\section{ACKNOWLEDGEMENTS}

Authors are sincerely grateful to Dr. Anders Hagborg (F) and Dr. Bill Buck (NY) for valuable suggestions and improvements as well as correcting of English in the manuscript, and to two anonymous reviewers for instructive comments that helped to improve the paper. The work was partially supported by the grants from the Russian Foundation for Basic Researches (13-04-00775, 15-34-20101) to VB.

\section{LITERATURE CITED}

Abramova, A.L. \& I.I. Abramov 1953a. Materials on hepatics of South Ossetia. Trudy Botanicheskogo Instituta im V.L. Komarova Akademii Nauk SSSR, Ser. 2, 8: 375-401 (in Russian). [Абрамова А.А., Абрамов И.И. 1953а. Материалы к печеночникам Южной Осетии // Труды ботанического института им. В.А. Комарова Академии наук СССР, сер. 2. Т. 8. С. 375-401].

Abramova, A.L. \& I.I. Abramov 1953b. On new and interesting species of mosses and hepatics from Caucasus. Botanicheskiye matertialy otdela sporovykh rastenij Botanicheskoho Institute imeni V.L. Komarova AN SSSR 9: 183-187 (in Russian). [Абрамова А.А., Абрамов И.И. 1953б. О новых и интересных видах мхов и печеночников с Кавказа // Ботанические материалы отдела споровых растений Ботанического института имени В.А. Комарова АН СССР Т. 9. C. 183-187].

Abramova, A.L. \& I.I. Abramov 1979. Rare and interesting species of mosses from Caucasus. Novosti Sistematiki Nizshikh Rastenii 16: 158-160 (in Russian). [Абрамова А.А., Абрамов И.И. 1979. Редкие и интересные виды мхов с Кавказа // Новости систематики низших растений T. 16. C. 158-160].

Abramov I.I., A.L. Abramova \& J. Duda 1963. To the liverwort flora of Caucasus. Lagodekhsky Reserve. Botanicheskive matertialy otdela sporovykh rastenij Botanicheskoho Institute imeni V.L. Komarova AN SSSR 16: 168-172 (in Russian). [Абрамов И.И., Абрамова А.А., Ауда Й. 1963. К флоре печеночников Кавказа. Аагодехский заповедник // Ботанические материалы отдела споровых растений Ботанического института имени В. $\Lambda$. Комарова АН CCCP T. 16. C. 168-172].

Abramov I.I., A.L. Abramova \& J. Duda 1964. To the liverwort flora of Caucasus (Bakuriani). Novosti Sistematiki
Nizshikh Rastenii 1: 275-280 (in Russian). [Абрамов И.И., Абрамова А.А., Ауда Й. 1964. К флоре печеночников Кавказа (Бакуриани) / / Новости систематики низших растений Т. 1. С. 275-280].

Abramov I.I., A.L. Abramova \& J. Duda 1966. To the liverwort flora of Caucasus (Adjara). Novosti Sistematiki Nizshikh Rastenii 3: 287-297 (in Russian) [Абрамов И.И., Абрамова А.А., Ауда Й. 1966. К флоре печеночников Кавказа (ААжария) // Новости систематики низших растений Т. 3. С. 287-297].

Abramov I.I., A.L. Abramova \& I.V. Radzimovskaya 1986. To the bryophyte flora of Abkhazia. Novosti Sistematiki Nizshikh Rastenii 23:191-196 (in Russian). [Абрамов И.И., Абрамова А.А., Радзимовская И.В. 1986. К бриофлоре Абхазии // Новости систематики низших растений T. 23. C. 191-196].

Bakalin, V.A. 2014a. Caucasian-Manchurian relationships in the hepatic flora. Arctoa 23:150-163.

Bakalin, V.A. 2014b. The study of type collection in Conservatoire et Jardin Botanique de la Ville de Genève $(G)$ : the hepatic genera Jungermannia, Solenostoma and Plectocolea. Arctoa 23:91-136.

Bakalin, V.A. \& K. Tigishvili 2013. Notes of Lophozia VII. On the distribution of some species of Lophozia in Georgia (Caucasus). Arctoa 22:121-123.

Bakalin, V.A., Z. Manvelidze, D. Kharazishvili \& N. Memiadze 2013. A small collection of hepatics from Adjara, Georgia. Arctoa 22:125-130.

Brotherus V.F. 1882. Enumeratio Muscorum Caucasi. Acta Societatis Scientarum Fennicae 19(12):1-170.

Bush, N.A. \& E.A. Bush 1936. Vegetation cover of eastern South Ossetia. Proizvoditelnyye sily Yugo-Osetii, V, AN SSSR, SSPS, serija Zakavkazskaya, Vol. 18. Moscow-Leningrad, 263 pp. (in Russian) [Буш Н.А., Буш Е.А. 1936. Растительный покров востока Южной Осетии // Производительные силы Юго-Осетии, 5, АН СССР, ССПС, серия Закавказская. Вып. 18. М. - $\Lambda .263$ с.].

Chikovani, N. 1972. New data on liverwort flora of Caucasus. In: Tesisy dokladov IV Zakavkazskogo soveshchaniya po sporovym rasteniyam. Erevan, pp. 83-85 (in Russian) [Чиковани Н. 1972. Новые сведения о флоре печеночников Кавказа. // Тезисы докладов 4 Закавказского совещания по споровым растениям. Ереван. С. 83-85].

Chikovani, N. 1973. New and rare hepatics for Caucasus. Novosti Sistematiki Nizshikh Rastenii 10: 284-285 (in Russian). [Чиковани Н. 1973. Новые и редкие печеночники Амя Кавказа // Новости систематики низших растений. Т. 10. С. 284-285].

Chikovani, N. 1978. To the history of study of hepatics in Caucasus. Vestnik Gruzinskogo Botanicheskogo obshchestva 7: 56-63 (in Russian). [Чиковани Н. 1978. К истории изучения печеночников Кавказа / / Вестник грузинского ботанического общества. Т. 7. С. 56-63].

Chikovani, N. 1986. Bryophyta. In: Flora sporovykh rastenii Gruqii. Tbilisi, Metsniereba, pp. 786-85 (in Russian). [Чиковани Н. 1978. Мохообразные // ФАора споровых растений Грузии. Тбилиси: Мецниереба. С. 786851].

Chikovani, N. \& T. Svanidze 2004. Checklist of bryophyte species of Georgia. Braun-Blanquetia 34:97-116.

Konstantinova, N.A., V.A. Bakalin, E.N. Andrejeva, A.G. Bezgodov, E.A. Borovichev, M.V. Dulin \& Yu.S. Mamontov 2009. Checklist of liverworts (Marchantiophyta) of Russia. Arctoa 18: 1-64.

Kuntze, O. \& E. Regel 1887. Plantae orientali-rossicae. Acta Horti Petropolitani 10(1):135-262. 
Bakalin et al.

Kürschner, H., K. Batsatsashvili \& G. Parolly 2013. Noteworthy additions to the bryophyte flora of Georgia. Herrogia 26(1): 213-216.

Ladyzhenskaya, K.I. 1951 On poorly known hepatic in the USSR flora Marchantia paleacea Bertol. Botanicheskiye materialy otdela sporovykh rastenii 7: 194-206 (in Russian). [Аадыженская К.И. 1951. О малоизвестном печеночнике во флоре СССР Marchantia paleacea Bertol. // Ботанические материалы отдела споровых растений Ботанического института имени В. $\Lambda$. Комарова АН CССР T. 7. C. 194-206].

Schljakov, R.N. 1981. Lophozia longiflora (Hepaticae) - a frequent component of plant communities. Botanicheskil Zhurnal 66(8):1197-1205 (in Russian). [ШАяков P.Н. 1981. Lophozia longiflora (Hepaticae) - частый компонент растительных сообществ // Ботанический журнац. T. 66, №. 8. C. 1197-1205].

Tigishvili, K. 2006. Ecocoenotic distribution of bryoflora in lower forest belt of Lagodekhi Reserve. Proceedings Georgian Academy Sciences. Biology Ser. B. 4: 61-66.

Váňa, J. 1970. Jungermannia caucasica sp.n. (Hepaticae). Preslia 42:96-97.

Váňa, J. 1976. Studien über die Jungermannioideae (Hepaticae) 10. Nardia. Folia Geobotanica et Phytotaxonomica 11: $367-425$.

Váňa, J. \& M.S. Ignatov 1990. Liverworts of Kholodnaya River (Abkhazia). Bulletin glavnogo botanicheskogo sada AN SSSR 56:43-44 (in Russian). [Ваня И., Игнатов M.C. 1990. Печеночники Холодной речки (Абхазия) // Бюлметень главного ботанического сада АН СССР. T. 56. C. 43-44].

Voronov, Yu. N. 1914. Materials to the liverwort flora of Caucasus. Iqvestiya Kavkazskogo Mureya 8: 81-88 (in Russian). Воронов Ю.Н. 1914. Материалы к флоре печеночников Кавказа // Известия Кавказского музея T. 8. C. 81-88]. 